\title{
Makorin rings the kisspeptin bell to signal pubertal initiation
}

\author{
Ali Abbara and Waljit S. Dhillo \\ Section of Endocrinology and Investigative Medicine, Department of Metabolism, Digestion and Reproduction, Imperial College London, Hammersmith Hospital, London, United Kingdom.
}

\begin{abstract}
The signals maintaining quiescence of the reproductive endocrine axis during childhood before its reawakening at puberty had been enigmatic. Studies in patients with abnormal puberty have illuminated the identity of the signals; kisspeptin has emerged as a major stimulator of puberty, and makorin RING finger protein 3 (MKRN3) as an inhibitory signal that prevents premature initiation of puberty. In this issue of the $J C I$, Abreu et al. investigated the mechanism by which MKRN3 regulates pubertal onset. The authors found that a reduction in MKRN3 alleviated the constraint on kisspeptin-expressing neurons to allow pubertal initiation, a phenomenon observed across species, including nonhuman primates. Further, the ubiquitinase activity of MKRN3 required its RINC finger domain, in order to repress the promoter activity of genes encoding kisspeptin and neurokinin B. These data advance our understanding of the regulation of kisspeptin-expressing neurons by MKRN3 to initiate puberty.
\end{abstract}

\section{What initiates puberty?}

Activation of the hypothalamic-pituitarygonadal (HPG) axis to initiate puberty and acquire competence for adult reproductive health is a fundamental physiological process. Conspicuously, the timing of the pubertal transition varies across the population and is contingent on a complex interplay between genetic, environmental, and metabolic factors (1). This genetic-environmental interplay is highlighted by a reduction in the age of pubertal onset over the last century, which has been attributed partly to metabolic factors such as obesity, as well as environmental factors such as endocrine disruptors (2-4).

However, puberty is not the first activation of the HPG axis during the lifespan; indeed, it is active in fetal and early neonatal life, termed "minipuberty." This early activation of the endocrine axis is believed to be important for priming reproductive organs to fully respond to gonadotropins during puberty. Minipuberty is especially important in boys, whereby the proliferation of immature Sertoli cells impacts the success of future spermatogenesis (3). Nevertheless, from the age of 6 months in boys and 3-4 years in girls, the HPG axis is inhibited and remains quiescent until its reactivation to initiate puberty $(2,3)$. However, what instigates this reactivation of the HPG axis has remained an intriguing mystery for many years; indeed, "What triggers puberty?" was one of the top unanswered questions in the scientific field published in 2005 (5). Since then, our knowledge of the key signals that initiate puberty has advanced substantially with some notable discoveries, of which MKRN3 is a further major breakthrough that sheds light on this mystery.

Conflict of interest: AA and WSD have undertaken consultancy work for Myovant Sciences Ltd. Copyright: () 2020, American Society for Clinical Investigation.

Reference information: / Clin Invest. 2020;130(8):3957-3960. https://doi.org/10.1172/JCI139586.

Key players in the neuroendocrine control of puberty

Revelation of the central actors responsible for pubertal initiation has predominantly emanated from studies in patients with disordered puberty, i.e., precocious (early) or delayed (late)/absent puberty. Many of these discoveries have not only transformed our understanding of the signals regulating puberty, but also more widely of the physiological regulation of the endocrine HPG axis.

In 2003, loss-of-function variants in the gene encoding the kisspeptin receptor were reported to result in a failure of pubertal maturation $(6,7)$. Conversely, activating variants of the kisspeptin receptor were found to cause central precocious puberty (CPP) $(8,9)$. Accordingly, hypothalamic kisspeptin expression is increased at the time of puberty, as is the number of kisspeptin-responsive gonadotropin-releasing hormone (GnRH) neurons (10). These data first indicated the crucial role of kisspeptin neurons as putative gatekeepers of pubertal maturation. Moreover, the function of kisspeptin extends far beyond its critical role in pubertal initiation to the regulation of hypothalamic GnRH function in both health and disease. Kisspeptin neurons integrate feedback from sex steroids and metabolic and nutritional signals to regulate GnRH neuronal secretion.

A second neuropeptide discovered in 2009 to play an important role in the pubertal transition is neurokinin $\mathrm{B}$, encoded by the TAC3 gene in humans (11). Studies in animal models have revealed that neurokinin B is coexpressed with kisspeptin and dynorphin in kisspeptin/neurokinin $\mathrm{B} /$ dynorphin (KNDy) neurons in the arcuate nucleus (12-15). These neuropeptides are believed to act in concert and in an autocrine manner to regulate the release of kisspeptin and thus determine the pulsatile nature of GnRH secretion. Consequently, KNDy neurons are regarded as vital components of the GnRH pulse generator. 


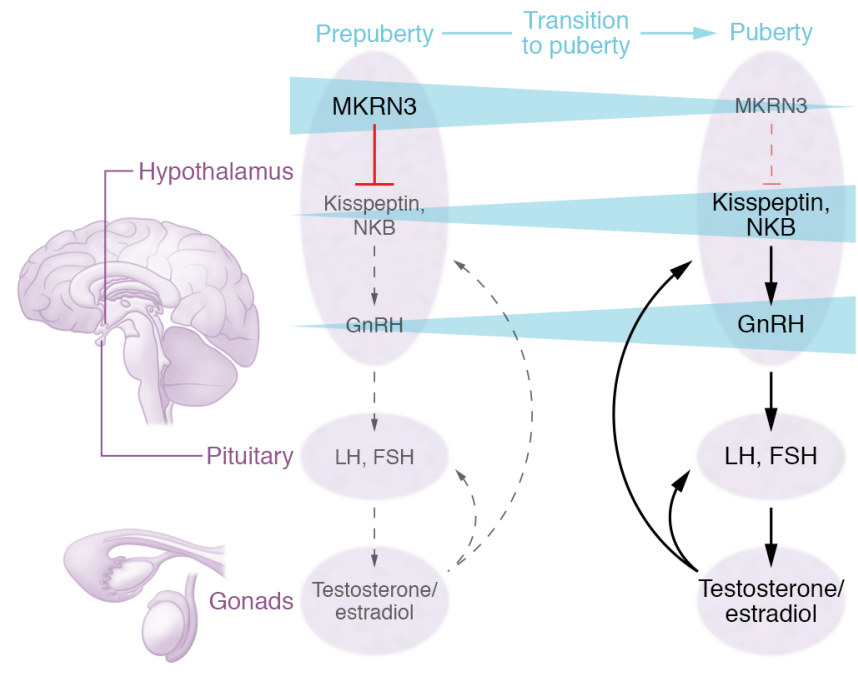

Figure 1. A model for how the transition to puberty is controlled by the effect of MKRN3 on kisspeptin-expressing neurons in the hypothalamus. Abreu et al. showed that MKRN3 repressed the promoter activity of genes encoding kisspeptin and neurokinin B (19). The reduction in MKRN3 in the lead up to puberty alleviates the constraint on kisspeptin/neurokinin B/dynorphin (KNDy) neurons to allow reactivation of the hypothalamic-pituitary-gonadal axis and the initiation of puberty. NKB, neurokinin $\mathrm{B}$; FSH, follicle-stimulating hormone.

Overall, activation of these KNDy neurons plays a key role in initiating puberty, but what constrains KNDy neuronal activity following minipuberty throughout childhood had remained an enigma.

In 2013, a landmark study reported that loss-of-function variants in the MKRN3 gene (previously known as ZNF127), which encodes makorin RING finger protein 3, resulted in CPP (16). Fittingly, RING in this context does not indicate that the protein has a circular structure but rather denotes really interesting new gene. Indeed, this was the first reported loss-of-function variant causing $\mathrm{CPP}$ and remains the commonest genetic cause of CPP, enabling the reclassification of many children previously labeled as having idiopathic CPP (17). A recent meta-analysis revealed that $M K R N 3$ variants are found in 9\% (95\%CI 4\%-15\%) of children with CPP, and more frequently in familial CPP (19\%) (18). Although typically CPP occurs more commonly in girls, $M K R N 3$ variants can cause CPP in both sexes, albeit more frequently in boys with CPP (22\% vs. $7 \%)$, and girls remain affected at an earlier age than boys (18).

MKRN3 is an intronless and maternally imprinted gene (i.e., only the paternal allele is expressed) located on chromosome 15q11.2 in the Prader-Willi syndrome (PWS) critical region. Gene imprinting refers to the epigenetic phenomenon that results in selective expression of genes depending on whether they are inherited from the maternal or paternal parent. For instance, while paternal inheritance of variants in the PWS critical region results in PWS (hypotonia, obesity, and hypogonadism), maternal inheritance of variants in the same region results in Angelman syndrome (seizures, developmental and neurological disorders). In a genome-wide association study to identify genes associated with the natural variation in pubertal onset, MKRN3 had the greatest association with menarche when paternally inherited (1).

\section{How does MKRN3 interact with kisspeptin neurons?}

Abreu and Kaiser published the seminal report in 2013 demonstrating that loss-offunction variants in MKRN3 cause CCP (2). In this issue of the JCI, Abreu and colleagues further investigate the mechanism by which $M K R N 3$ constrains pubertal maturation (19). They demonstrated that MKRN3 was expressed at higher levels in the hypothalamus than the liver or testes, consistent with its role as an inhibitor of hypothalamic kisspeptin neurons. Further, they confirmed the presence of MKRN3 across species with expression in both the rat and nonhuman primate medial basal hypothalamus (19), consistent with its central role in the neuroendocrine control of puberty. More specifically, though MKRN3 was expressed in a number of hypothalamic nuclei, it was most strongly expressed in the arcuate and ventromedial nuclei (19). Moreover, MKRN3 colocalized with kisspeptin-expressing neurons, and its expression decreased during the lead up to puberty (19), consistent with the working hypothesis that the reduction in MKRN3 alleviates the constraint on these neurons to allow the initiation of puberty (Figure 1). The investigators revealed further detail as to how MKRN3 interacted with KNDy neurons, specifically that MKRN3 repressed the promoter activity of genes encoding kisspeptin and neurokinin B (19). Finally, the authors demonstrated that MKRN3 achieved inhibition of KNDy neurons through its action as an E3 ubiquitin ligase. Ubiquitin is a small regulatory protein, which as its name suggests, is ubiquitously present in most eukaryotic cells. Ubiquitination refers to the process by which ubiquitin binds to a substrate protein to modify its function, or to target it for degradation. Ubiquitination involves 3 sequential steps that are catalyzed by specific groups of enzymes, namely activation (E1), conjugation (E2), and ligation (E3). E3 enzymes can also undergo autoubiquitination, whereby they catalyze the addition of polyubiquitin to themselves, a feature that can be used to monitor E3 enzymatic activity. Abreu et al. demonstrated that the RING finger domain of MKRN3 was obligatory for its ubiquitinase activity, which in turn was required to repress kisspeptin and neurokinin $\mathrm{B}$ gene promoters (19). MKRN3 is an E3 ubiquitin ligase, and methyl-CpG-DNA binding protein (MBD3) has recently been identified as a substrate for its E3 ligase activity (20). Additionally, MKRN3-mediated ubiquitination has been reported to disrupt interactions between MBD3 and the GNRH1 promoter to cause epigenetic silencing of GNRH1 expression (which encodes the precursor of GnRH) (20). However, this action may not be exclusive, as MKRN3 has been shown to associate with 81 protein interaction partners, of which 21 are implicated in the determination of pubertal onset (21).

Data from other studies corroborate the Abreu et al. (15) hypothesis that the decline in MKRN3 acts as a signal to initiate puberty. Circulating levels of MKRN3 
decline both before and during puberty, reducing by almost half across the pubertal transition in boys (22); the fall in circulating MKRN3 levels appears to occur most precipitously in early puberty and inversely correlates with increases in luteinizing hormone (LH) and testosterone (23). Circulating MKRN3 levels are lower in girls with CPP than healthy controls (24-26), although tellingly, MKRN3 levels fall rather than rise after $\mathrm{GnRH}$ agonist treatment of girls with CPP $(27,28)$. Men with congenital hypogonadotropic hypogonadism had similar circulating levels of MKRN3 to those of healthy men (29).

Of course, while the discovery of MKRN3 signals a new chapter in the story unravelling the neuroendocrine control of puberty, many pages have yet to be written. Recent supportive evidence demonstrates that puberty is accelerated in an Mkrn3-knockout mouse, which has increased hypothalamic expression of Gnrh1, although, notably, no change in the expression of genes encoding kisspeptin or neurokinin B was identified (20). Moreover, the microRNA (short noncoding RNA that represses posttranscriptional gene expression), miR-30, can repress MKRN3 expression and thus could mediate the signal that releases the MKRN3 constraint on KNDy neurons to initiate puberty (30). Sex steroids have been prescribed clinically to induce pubertal maturation in children with delayed puberty. Although Abreu et al. found that sex steroids failed to change $M K R N 3$ expression (19), estrogen exposure in rats has been shown to suppress miR-30 expression, thus suggesting an indirect mechanism by which estrogen could affect $M K R N 3$ expression (30).

\section{Future directions}

In future studies, it would be intriguing to investigate the expression of MKRN3 at around the time of minipuberty to ascertain whether corresponding changes in MKRN3 are apparent. Additionally, it would be interesting to discern whether MKRN3 plays any important role after puberty and whether the system is reversible (i.e., can overexpression of $M K R N 3$ in adult animals switch off the HPG axis once activated?). Further work is required to investigate whether MKRN3 mediates the impact of metabolic factors on pubertal onset. Interestingly, MKRN3 levels correlate with HBA1c in healthy men (29) and Mkrn3-knockout mice had subtle sex-specific alterations in body weight (20). These data, coupled with the interaction of MBD3 with ten-eleven translocation methylcytosine dioxygenase 2 (TET2) (20) (a DNA methylase reported to be a substrate for AMP-activated kinase, AMPK), suggest that further study is warranted to investigate how metabolic factors interact with MKRN3, perhaps via epigenetic changes, to impact pubertal onset. A clear candidate for mediating the effects of body weight on pubertal initiation is leptin; however, the effects of leptin on pubertal initiation appear to be independent of MKRN3 (31). Thus, further work to identify the factors that regulate MKRN3 represents an intriguing area of active research.

In conclusion, the landmark discovery of MKRN3 considerably advances our understanding of the signals that regulate the normal timing of puberty and highlights the relevance of epigenetic changes to the neuroendocrine control of puberty.

\section{Acknowledgments}

The Section of Endocrinology and Investigative Medicine was funded by grants from the Medical Research Council (MRC) and the National Institute for Health Research (NIHR), and was supported by the NIHR Biomedical Research Centre Funding Scheme. The views expressed are those of the authors and not necessarily those of the MRC, BBSRC, the NHS, the NIHR, or the Department of Health. AA was supported by NIHR Clinician Scientist Award CS-2018-18-ST2-002. WSD was supported by an NIHR Research Professorship, NIHR-RP-2014-05-001.

Address correspondence to: Waljit S. Dhillo, Section of Endocrinology and Investigative Medicine, Imperial College London, 6th Floor, Commonwealth Building, Hammersmith Hospital, Du Cane Road, London, W12 ONN, United Kingdom. Phone: 44.208.383.3242; Email:w.dhillo@ imperial.ac.uk.

1. Perry JR, et al. Parent-of-origin-specific allelic associations among 106 genomic loci for age at menarche. Nature. 2014;514(7520):92-97.

2. Abreu AP, Kaiser UB. Pubertal development and regulation. Lancet Diabetes Endocrinol. 2016;4(3):254-264
3. Young J, et al. Clinical management of congenital hypogonadotropic hypogonadism. Endocr Rev. 2019;40(2):669-710.

4. Sørensen K, Aksglaede L, Petersen JH, Juul A. Recent changes in pubertal timing in healthy Danish boys: associations with body mass index. J Clin Endocrinol Metab. 2010;95(1):263-270.

5. [No authors listed]. So much more to know. Science. 2005;309(5731):78-102.

6. de Roux N, Genin E, Carel JC, Matsuda F, Chaussain JL, Milgrom E. Hypogonadotropic hypogonadism due to loss of function of the KiSS1-derived peptide receptor GPR54. Proc Natl Acad Sci U S A. 2003;100(19):10972-10976.

7. Seminara SB, et al. The GPR54 gene as a regulator of puberty. $N$ Engl J Med. 2003;349(17):1614-1627.

8. Teles MG, et al. A GPR54-activating mutation in a patient with central precocious puberty. $N \mathrm{Engl}$ JMed. 2008;358(7):709-715

9. Silveira LG, et al. Mutations of the KISS1 gene in disorders of puberty. J Clin Endocrinol Metab. 2010;95(5):2276-2280.

10. Herbison AE. Control of puberty onset and fertility by gonadotropin-releasing hormone neurons. Nat Rev Endocrinol. 2016;12(8):452-466.

11. Topaloglu AK, et al. TAC3 and TACR3 mutations in familial hypogonadotropic hypogonadism reveal a key role for neurokinin $\mathrm{B}$ in the central control of reproduction. Nat Genet 2009;41(3):354-358.

12. Moore AM, Coolen LM, Lehman MN. Kisspeptin/Neurokinin B/Dynorphin (KNDy) cells as integrators of diverse internal and external cues: evidence from viral-based monosynaptic tract-tracing in mice. Sci Rep. 2019;9(1):14768.

13. Moore AM, Coolen LM, Porter DT, Goodman RL, Lehman MN. KNDy cells revisited. Endocrinology. 2018;159(9):3219-3234.

14. Weems P, Smith J, Clarke IJ, Coolen LM, Goodman RL, Lehman MN. Effects of season and estradiol on KNDy neuron peptides, colocalization with D2 dopamine receptors, and dopaminergic inputs in the ewe. Endocrinology. 2017;158(4):831-841.

15. Mittelman-Smith MA, Krajewski-Hall SJ, McMullen NT, Rance NE. Ablation of KNDy neurons results in hypogonadotropic hypogonadism and amplifies the steroid-induced LH surge in female rats. Endocrinology. 2016;157(5):2015-2027.

16. Markic J, et al. CD15s is a potential biomarker of serious bacterial infection in infants admitted to hospital. Eur J Pediatr. 2013;172(10):1363-1369.

17. Bessa DS, et al. High frequency of MKRN3 mutations in male central precocious puberty previously classified as idiopathic. Neuroendocrinology. 2017;105(1):17-25.

18. Valadares LP, et al. MKRN3 mutations in central precocious puberty: a systematic review and meta-analysis. JEndocr Soc. 2019;3(5):979-995.

19. Abreu AP, et al. MKRN3 inhibits the reproductive axis through actions in kisspeptin-expressing neurons. J Clin Invest. 2020;130(8):4486-4500.

20. Li C, et al. MKRN3 regulates the epigenetic switch of mammalian puberty via ubiquitination of MBD3. Nat Sci Rev. 2020;7(3):671-685.

21. Yellapragada V, et al. MKRN3 interacts with several proteins implicated in puberty timing 
but does not influence GNRH1 expression. Front Endocrinol (Lausanne). 2019;10:48.

22. Busch AS, Hagen CP, Almstrup K, Juul A. Circulating MKRN3 levels decline during puberty in healthy boys. J Clin Endocrinol Metab. 2016;101(6):2588-2593.

23. Varimo T, Dunkel L, Vaaralahti K, Miettinen PJ, Hero M, Raivio T. Circulating makorin ring finger protein 3 levels decline in boys before the clinical onset of puberty. Eur JEndocrinol. 2016;174(6):785-790.

24. Grandone A, et al. MKRN3 levels in girls with central precocious puberty and correlation with sexual hormone levels: a pilot study. Endocrine. 2018;59(1):203-208.

25. Hagen CP, Sørensen K, Mieritz MG, Johannsen
TH, Almstrup K, Juul A. Circulating MKRN3 levels decline prior to pubertal onset and through puberty: a longitudinal study of healthy girls. JClin Endocrinol Metab. 2015;100(5):1920-1926.

26. Ge W, Wang HL, Shao HJ, Liu HW, Xu RY. Evaluation of serum makorin ring finger protein 3 (MKRN3) levels in girls with idiopathic central precocious puberty and premature thelarche. Physiol Res. 2020;69(1):127-133.

27. Jeong HR, Lee HJ, Shim YS, Kang MJ, Yang S, Hwang IT. Serum Makorin ring finger protein 3 values for predicting central precocious puberty in girls. Gynecol Endocrinol. 2019;35(8):732-736.

28. Grandone A, et al. MKRN3 levels in girls with central precocious puberty during GnRHa treatment: a longitudinal study. Horm Res Paediatr.
2018;90(3):190-195.

29. Varimo T, Hero M, Känsäkoski J, Vaaralahti K, Matikainen N, Raivio T. Circulating makorin ring-finger protein-3 (MKRN3) levels in healthy men and in men with hypogonadotropic hypogonadism. Clin Endocrinol (Oxf). 2016;84(1):151-152.

30. Heras V, et al. Hypothalamic miR-30 regulates puberty onset via repression of the puberty-suppressing factor, Mkrn3. PLoS Biol. 2019;17(11):e3000532.

31. Roberts SA, et al. The peripubertal decline in Makorin ring finger protein 3 expression is independent of leptin action [published online May 21, 2020]. J Endocr Soc. https://doi.org/10.1210/ jendso/bvaa059. 